\title{
Numerical Evolution of Single, Binary and Triple Stars
}

\author{
Peter P. Eggleton ${ }^{1,2}$ \\ ${ }^{1}$ Institute of Geophysics and Planetary Physics \\ ${ }^{2}$ Physics and Allied Technologies Division \\ Lawrence Livermore National Laboratory, 7000 East Ave, \\ Livermore, CA94551, USA \\ email: ppe@igpp.ucllnl.org
}

\begin{abstract}
I discuss my stellar evolution code Ev in the context of simulations of large clusters of stars. It has long been able to handle single stars, and also binary stars up to a point. That point is far beyond what other codes are able to do, but well short of what is necessary for believable simulations. A recent version, Ev(Twin), can in principle deal with the contact phase of binary evolution, but it is not yet clear what the physical interaction is that needs to be simulated.

An upgrade, which I hope will be only a few lines, should allow it to follow Kozai cycles with tidal friction, a process that strongly influences the orbital period of close pairs that reside within wide, non-coplanar triples. However, there are many substantial gaps in the physics of even single stars, let alone binaries or triples.
\end{abstract}

Keywords. stars: evolution, stars: binaries: general

\section{Introduction}

There is quite a lot that we seem to understand reasonably well about single and binary stars, but also quite a lot that we clearly don't understand and so we ought to try harder. For many people, a stellar evolution code is something you take off the shelf, turn into a module, and plug into some larger computational entity. For me, a stellar evolution code is a warm, living entity, a small child that needs constant nurturing and encouragement in the hope that it will grow to a comfortable and productive adulthood. It should develop the social skills to interact with other codes, but it should also develop its brain. This must be sufficiently supple that new ideas can be grafted in without major rearrangement.

The main difference between a code and a child is that a code does exactly what you tell it. This has disadvantages as well as advantages.

I call my code Ev. I run it usually in one of three operational modes that I call Ev(Single), Ev(Flip-Flop) and Ev(Twin). The first deals with single stars, the second with binaries under the assumption that Roche-Lobe overflow (RLOF) is the main interaction between them, and is reasonably (but not necessarily wholly) conservative both of total mass and of orbital angular momentum. Flip-Flop refers to the fact that it will advance the primary (' $* 1$ ') say 200 timesteps, then the secondary $\left({ }^{\prime} * 2\right.$ ') until it catches up, and then advances $* 1$ another 200 timesteps, and so on. The reason for this is a small economy: very often when we follow $* 2$ in a rather close binary we find that it fills its own Roche lobe while $* 1$ still does. It evolves into contact, in other words, and there is no point in following $* 1$ through to a supernova (SN) explosion if in practice $* 2$ evolves into contact with $* 1$ quite early in $* 1$ 's evolution. Almost certainly the fact of 

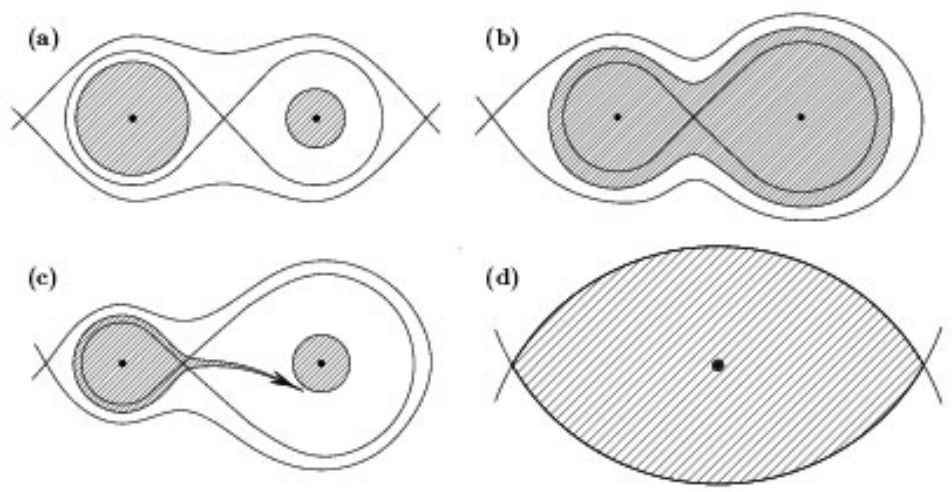

Figure 1. (a) A detached binary, also showing the critical inner (and outer) Lagrangian surface. (c) A semidetached system, with mass flowing from the loser to the gainer. The components have equal mass but different radii, perhaps as a result of differential stellar wind. (b) The gainer expands to fill its own Roche lobe, leading to contact. (d) Progressive mass transfer reduces one component to zero, and the other is then a rapidly rotating single star.

contact makes the later evolution of $* 1$ obsolete - we need some different approach to compute the effect of contact. Nelson \& Eggleton (2001) showed that contact is almost inevitable at a late stage in the evolution of short-period binaries; it is avoided only if the masses are fairly nearly equal, and the period is not too short. Fig. 1 is a cartoon of the progression of a binary from an initial detached configuration through a semidetached configuration, then into contact and ultimately (in many cases, I expect) into a merged single, rapidly-rotating star.

I believe that contact binaries are the proverbial elephant in the drawing room, which everyone prefers to ignore because they don't know what to do about it.

Ev(Twin) is intended to be the new process that will solve the contact problem, but it is still in development. It has in fact solved just one contact binary so far, which I will show shortly.

Even Ev (Single) contains some very powerful and original concepts, of which the main one is that equations for all of (a) the structure, (b) the composition, and (c) the adaptive mesh, are solved simultaneously and implicitly. Because of this concept, Ev(Flip-Flop) is enabled to be very powerful also. In order to deal with RLOF in a binary, it differs from the Single mode in one significant line only: the boundary condition $M_{1}=$ given is replaced by one which says that $-d M_{1} / d t$ is proportional to the cube of $\ln R_{1} / R_{\text {lobe, } 1}$, provided this is positive, when doing $* 1$, and by $d M_{2} / d t=-d M_{1} / d t$ when doing $* 2$. This is based on the approximination of Paczyński \& Sienkiewicz (1972), itself based on a Bernoulli-type approximation to the fluid flow involved in RLOF.

Flip-Flop can incorporate what I call 'partially non-conservative' effects, such as stellar winds, magnetic braking, tidal friction, circularistion of eccentric orbits, and synchronism of non-corotating components, but only for $* 1$. The wind from $* 2$, for example, will depend on parameters relating to $* 2$ such as its radius, luminosity and rotation rate. These will not be known at the time that one is advancing the evolution of $* 1$. 
(a) Theoretical HRD

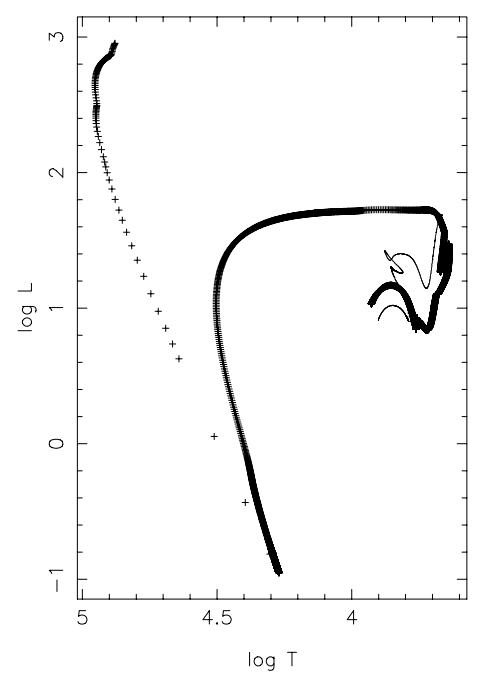

(b) e, $P, P_{R 1}, P_{R 2}$

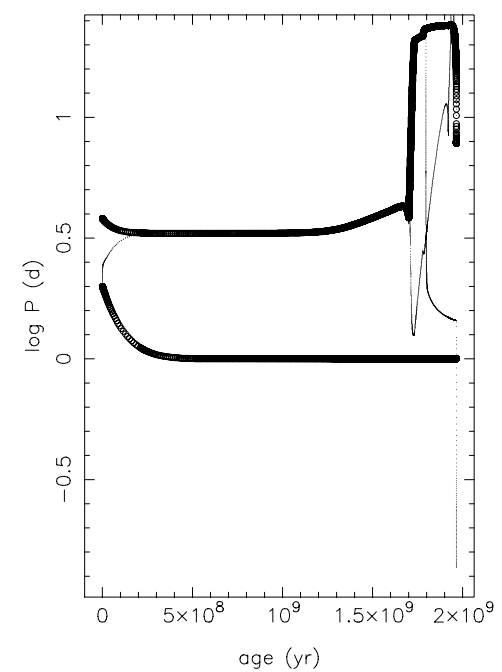

Figure 2. Evolution of a system like Z Her, starting with $\left(1.8+1.68 M_{\odot} ; 3.8 \mathrm{~d}, e=0.3\right)$, using Ev(Twin). (a) Theoretical HRD: thick line $* 1$, thin line $* 2$. The evolution terminated with a nova explosion on $* 1$, following reverse RLOF. (b) Eccentricity (lower thick line), orbital period (upper thick line), and both rotational periods (thin lines) as functions of age. The rotational period of $* 1$ ends shorter than that of $* 2$.

Ev(Twin) is revolutionary in that it deals with this and several other problems by solving both of the components simultaneously. Actually it is not quite revolutionary: Robertson \& Eggleton (1977) did this. But in 1977 big, powerful computers had only $50 \mathrm{~KB}(\mathrm{~KB}$ !) of random-access memory, and it was quite a chore to squeeze in a matrix that was $2 \times 2$ times as large as the usual one. But the virtue of Twin is, or ought to be, that it can deal numerically with the contact phase. Contact involves what I would like to call a 'strong interaction', ie. the behaviour of one component is very strongly influenced by what the other component is doing at the same time. The obvious answer to this is to enlarge the concepts of 'simultaneous' and 'implicit' to the whole binary system.

Ev (Twin) can deal well with systems that are not in contact, but where non-conservative processes in both components are happening simultaneously. Fig. 2 shows the evolution of a system starting with parameters $\left(1.8+1.68 M_{\odot} ; 3.8 \mathrm{~d}, e=0.3\right)$. It is strikingly different from what would go on in presumed conservative evolution, but space does not permit a detailed description.

The difficulty with contact binaries is to think of a reasonable mathematical/physical model of what goes on in contact. On the one hand mass can flow, in either direction in principle, between the outer layers of one component and the outer layers of the other, all the way down to the inner Lagrangian surface. On the other hand, heat can also flow. The direction of heat flow might change with depth, as might the direction of mass flow.

My best shot so far treats the heat flow by a model based on the fact that, in the Sun, it is well known (though not yet well understood) that there is differential rotation. A surface equatorial belt, about $30 \%$ of the depth of the Sun and about $\pm 30^{\circ}$ in breadth, rotates about $10 \%$ faster than the mean. In a frame that rotates with the mean Sun, this belt is carrying a colossal flow of heat 'sideways'. Work it out: it's about $3000 L_{\odot}$. Of course this makes no difference to the Sun, because the Sun is roughly axially symmetric. But in a contact binary consisting of say $1+0.5 M_{\odot}$ components which share a single 
(a) $M_{1}, M_{2}$

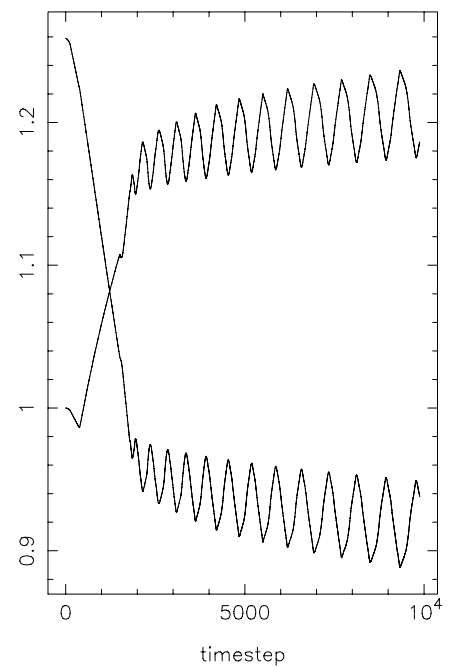

(b) $L_{1}, L_{2}, \Delta L, 10 \Delta \log T$

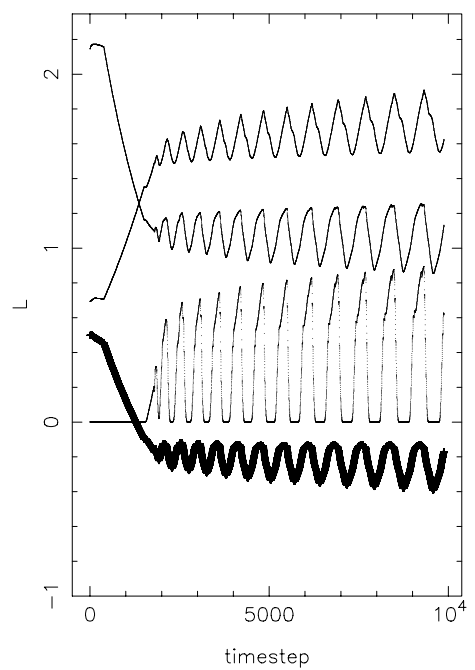

Figure 3. (a) $M_{1}$ and $M_{2}$ plotted against timestep number. In actual time, the oscillatory stretch is only about $2 \%$ of the entire evolution. (b) Evolution of $L_{1}, L_{2}$ (upper two curves), $\Delta L$, and $10 \Delta \log T$ (thick curve) against timestep number, in a binary which evolves from detached through semidetached to early contact. $\Delta L$ is the amount of luminosity transferred in contact, and so is zero during a detached or semidetached phase. $\Delta \log T$ is the difference in effective temperatures of the two components.

envelope that accounts for the outer $5-10 \%$ of each by radius, even $0.1 \%$ of this flux, flowing largely around the equatorial belt of one star and on around the equatorial belt of the other, and on further, would represent a large exchange of luminosity between the components, quite possibly equalising, more or less, the two surface temperatures as is normally observed in contact binaries.

Fig. 3 is an attempt (Yakut \& Eggleton 2005) to model part of this with Ev(Twin). Fig 3a shows the two masses, and the upper two curves of Fig $3 \mathrm{~b}$ the two luminosities. $M_{1}$ and $L_{1}$ started to decrease rapidly at RLOF, but shortly after the mass and luminosity ratio reversed the system came into contact. There followed a series of oscillations, but with a slight trend discernible: the mass ratio continued to depart from unity, on average. The oscillations are rather rapid, and demand small timesteps; the 11 oscillations occupy about $2 \%$ of the entire evolutionary sequence.

It is not clear how real these oscillations are, although similar oscillations were seen (using semi-analytical techniques) by Lucy (1976) and Flannery (1976), as well as by Robertson \& Eggleton (1977). They appear to contradict the observational datum that the great majority of contact binaries have closely equal temperatures. The thick line of Fig $3 \mathrm{~b}(10 \Delta \log T)$ oscillates between -0.1 and -0.4 ; the first value is acceptable, but the second is not. However, there are a few arguments that suggest that the inconsistency may be more apparent than real:

(i) The model presented here has a mass ratio rather close to unity - of necessity, since I have had difficulty getting the code to work for more extreme mass ratios - and so is not representative of real contact binaries, whose mass ratios are usually in the range $2-10$

(ii) There does exist a small population of close binaries that have similar periods to contact binaries, but rather more unequal temperatures. They are sometimes called 
'near-contact binaries'. There is perhaps one of these for every 10-20 normal contact binaries, but the statistics are very poor

(iii) We might hypothesise that as the mass ratio gets more extreme, the time spent with the temperatures substantially different becomes a smaller fraction of the time spent with temperatures nearly equal.

Models in contact have so far turned out to be very expensive of computational time. But I hope to produce some more, with more extreme mass ratios, in due course. However it is possible that some important element of the physics is missing, and that when this is included the evolution will actually be simpler to calculate. Perhaps there is some kind of 'thermal inertia' which turns the oscillatory behaviour into monotonic behaviour; but most attempts to fudge that lead to the two components having permanently different temperatures, rather than permanently equal temperatures.

I believe, or at least hope, that it will not be very difficult to add in the equations that govern Kozai cycles. These are cycles of eccentricity, but not semimajor axis or period, that are induced in a binary that is part of a triple system where the outer orbit is inclined at more than $39^{\circ}$ to the inner orbit. When tidal friction is included (which it already is) the inner orbit does suffer a reduction in period and semimajor axis: see the poster by Kisseleva-Eggleton \& Eggleton in this conference. We believe this may be vital in producing the shortest-period systems.

I and my colleagues at Lawrence Livermore Laboratory hope to gain insight into the physics of contact binaries, tidal friction and several other evolutionary problems, by modeling stars in 3 dimensions with the code Djehuty; see Dearborn et al. (2006) and Eggleton et al. (2006).

\section{Conclusion}

When evolving a cluster, the evolution of the single stars (and of wide binaries, if any) can be well approximated by interpolating in a pre-computed grid of, say, 1000 single stars with a range of masses. But binaries have too many parameters to be pre-computed. A code like Ev(Twin) will be needed for parallelised evolution, but further development of the contact model is necessary.

\section{Acknowledgements}

This work performed under the auspices of the U.S. Department of Energy by Lawrence Livermore National Laboratory under Contract DE-AC52-07NA27344.

\section{References}

Dearborn, D. S. P., Eggleton, P. P., \& Lattanzio, J. C. 2006, ApJ, 639, 405

Eggleton, P. P., Dearborn, D. S. P., \& Lattanzio, J. C. 2006, Science, 314, 1580

Flannery, B. P. 1976, ApJ, 205, 217

Lucy, L. B. 1976, ApJ, 205, 208

Nelson, C. A. \& Eggleton, P. P. 2001, ApJ, 552, 664

Paczyński, B. \& Sienkiewicz, R. 1972, A\&\&A, 22, 73

Robertson, J. A. \& Eggleton, P. P. 1977, MNRAS 179, 359

Yakut, K. \& Eggleton, P. P. 2005, ApJ, 629, 1055 